\title{
The Impact of Linguistic and Cultural Congruity on Persuasion by Conversational Agents
}

\author{
Langxuan Yin ${ }^{1}$, Timothy Bickmore ${ }^{1}$, Dharma E. Cortés ${ }^{2}$ \\ ${ }^{1}$ Northeastern University \\ \{yinlx, bickmore\}@ccs.neu.edu \\ ${ }^{2}$ Harvard Medical School \\ dharma_cortes@hms.harvard.edu
}

\begin{abstract}
We present an empirical study on the impact of linguistic and cultural tailoring of a conversational agent on its ability to change user attitudes. We designed two bilingual (English and Spanish) conversational agents to resemble members of two distinct cultures (Anglo-American and Latino) and conducted the study with participants from the two corresponding populations. Our results show that cultural tailoring and participants' personality traits have a significant interaction effect on the agent's persuasiveness and perceived trustworthiness.
\end{abstract}

Keywords: agent, language, culture, persuasion

\section{Introduction}

There has been a growing interest in developing conversational agents that can motivate users to change their health behavior. In the health care domain, several studies have now demonstrated that tailored messages - individualized to various aspects of users, such as demographic, personality, or lifestyle variables-are more persuasive and effective at achieving health behavior change [1], and the more variables that messages are tailored on, the more effective they are.

According to the Elaboration Likelihood Model, one of the leading theoretical frameworks in persuasion research, individuals are predisposed to processing messages through one of two routes to persuasion: a central route, where an individual considers a message via effortful, logical thinking, and the peripheral route, where the decision making is more reliant on heuristic environmental cues of the message, sender, and context [2], a trait referred to as "need for cognition" [3]. Message tailoring is thought to impact both routes to persuasion [1].

One form of tailoring that has received recent attention in the virtual agents community is adaptation of an agent's apparent culture. However, much of the existing research on this topic has only investigated whether or not users could perceive the cultural manipulations, but not the effect of these manipulations on outcomes of practical significance. A related form of tailoring is whether an agent speaks to users in their preferred (L1) natural language, or in a second (L2) language, assuming users are bilingual. Message tailoring theory would predict that the effects 
of cultural tailoring and linguistic tailoring should be separate and additive. However, a study by Luna, et al, on the effects of linguistic and cultural tailoring of websites on product evaluations demonstrated an interaction between these two factors, which the authors explained using the Elaboration Likelihood Model and differences in message processing effort between L1 and L2 text [4].

In the current study, we explore the separate and relative effects of cultural and linguistic tailoring of a conversational agent on its ability to change user attitudes. In order to inform the design of future counseling agents, we are interested in determining which of these manipulations are more effective, and what role personality traits such as need for cognition play in this process.

\section{Related Work}

Earlier research on virtual agents has shown a tendency of users' preference of culturally tailored agents. Rossen et al., using virtual agents with different skin tones, found that Caucasian subjects tend to show more empathy to the agent with a lighter skin tone [5]. Endrass et al. showed that German subjects preferred virtual characters speaking in the German way to those speaking in the Japanese way (in terms of silence and speech overlapping, etc.) [6], and similar preference tendencies were found among American and Arabic subjects as well [7]. Other studies such as that conducted by Jan et al. also suggest that people tend to favor a virtual agent resembling an in-group member of their culture [8].

Virtual agents have been shown to be effective in persuasion as well. By delivering persuasive messages via humans and virtual characters, Zanbaka et al. concluded that virtual characters can be as effective as humans at persuasion [9]. A study by Katagiri et al. has shown that virtual agents engaged in relationships with their users and other agents are effective in changing user's behaviors [10]. Also, in Schulman and Bickmore's experiment, participants had significantly more positive attitudes towards exercise after interacting with an exercise counseling virtual agent [11].

\section{Experiment Design}

To determine the effects of linguistic and cultural tailoring of a conversational agent on persuasion, we conducted a $2 \times 2$ factorial between-subjects experiment. Independent variables are linguistic congruity (whether the agent speaks the language that is L1 or L2 for a subject) and cultural congruity (high or low, relative to a subject's cultural affiliation).

The experiment protocol was similar to that from Schulman and Bickmore [11], inspired by the desert survival problem, commonly used to measure persuasion [12]. Participants performed a ranking task designed to measure attitudes towards exercise, then held a conversation with an agent, which consisted of a social dialogue and a persuasive dialogue. Finally, participants completed the same ranking task again, and perceptions of the agent were assessed. 


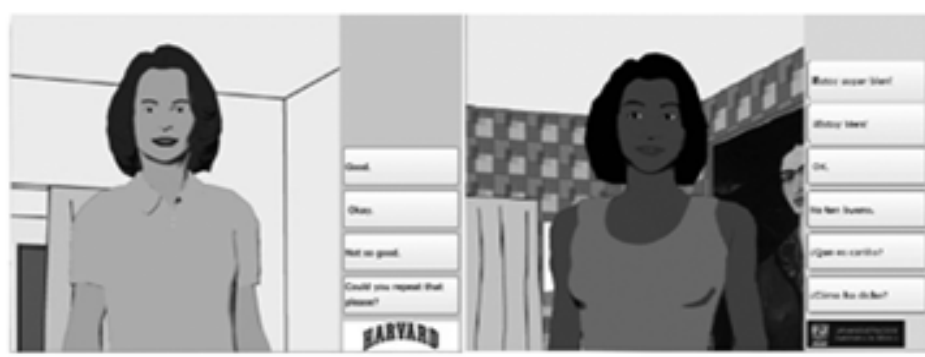

Fig. 1. Katherine (left), designed to resemble a member of the Anglo-American culture, and Catalina (right), designed to resemble a member of the Latino culture.

Anglo-American and Latina Agents. Two agents were developed for this study (Fig. 1). Katherine (the "Anglo-American" agent) was designed to resemble an Anglo-American in appearance, behavior and values, and was situated in a background picturing an Anglo-American-looking household. Catalina (the "Latina" agent) was designed to resemble a member of the Latino culture and was situated in a Latino-looking household background.

At the beginning of an interaction, the agent walked on to the screen and greeted the user, and then she talked about her personal background, which differed based on the agent's simulated culture. Following this, the agent presented a persuasive dialogue similar to that used by Schulman and Bickmore [11], arguing for the benefits of regular exercise and against statements about the disadvantages of regular exercise. However, the two agents argued for and against the same statements in different ways: the Anglo-American agent focused on participants' well-being, whereas the Latina agent expressed more interest in participants' family and friends, due to the importance of these aspects in the Latino culture [13]. A short clip of music representing the agent's culture was played as the agent walked on the screen. The Latina agent was also shown at a closer proximity to the user more often than the Anglo-American agent, because Latinos "interact at closer distances and employ more touching behaviors when interacting with family and friends" [14].

Linguistic Congruity. All dialogue content was first developed in English, and then translated to Spanish by a bilingual research assistant (native Spanish speaker). The Spanish scripts were then reviewed for accuracy by a native Spanish speaking researcher in our research team. The scripts were processed by a text-to-speech engine at runtime, using a female English voice (Loquendo "Susan") and a female Spanish voice (Loquendo "Soledad").

Measures. Four 7-point scale Likert questions were used as a manipulation check for cultural congruity: "How much do you feel that the agent is a member of the American culture", "How much do you feel that the agent is a member of the Latino/Hispanic culture", "How easy was it to understand the agent's language" and "How much do you feel you and the agent are from the same culture".

The ranking task consisted of a list of ten statements about regular exercise from most to least important (see Figure 2), and a score ranging from 1 (least important) to 10 (most important) was used to create a composite measure, subtracting the total 
score of the items argued against from the total score of the items argued for. The difference between the two measures before and after the conversation was calculated to measure persuasion (following Schulman \& Bickmore [11]).

Need for cognition was measured using the Need for Cognition Scale short form [2]. A high need for cognition score indicates the participant is predisposed to being influenced by central route to persuasion, while a low score indicates the participant is predisposed to being influenced by peripheral cues. And also, agent trustworthiness was measured by a standardized trust scale [15].

A semi-structured interview was conducted at the end of the study, focused on the user's opinions on the agent's cultural background and on their interaction.

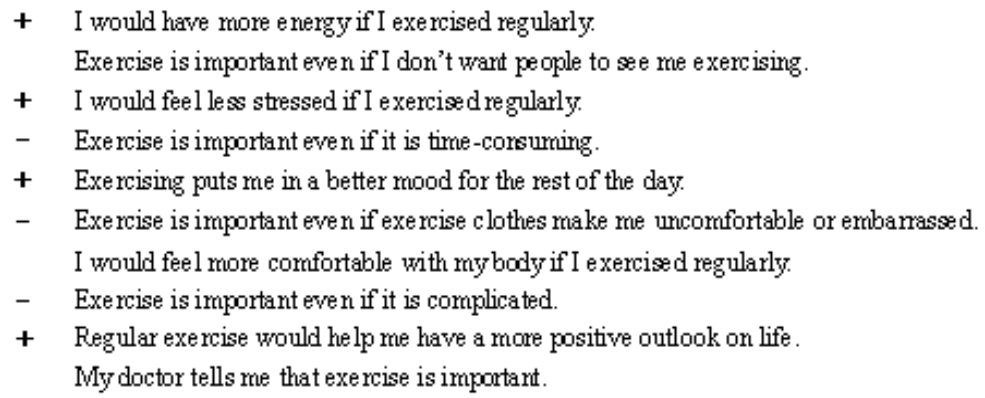

Fig. 2. Ten statements about regular exercise. Statements starting with a "+" are those that the agent argued for, and those starting with a "-" are those argued against. Statements starting with neither were not mentioned.

\section{Results}

Participants. Participants were recruited via fliers and advertisements, and enrolled only if they were able to speak read and understand both English and Spanish fluently and were either: a) an Anglo-American born in the United States who had stayed in Spanish-speaking Latin American countries for at least two months with English being their first language, or b) a Latino/Latina born in a Spanish-speaking Latin American country who had lived in the United States for at least two months, with Spanish being their first language.

Among the 43 participants recruited for the study, $65.0 \%$ were Latinos, and $44.2 \%$ were female. Participants' ages ranged from 18 to 65 , and over half of them (65.0\%) had been doing regular physical activity for more than 6 months.

Manipulation Checks. MANOVA tests reveal that the Latina agent was perceived as significantly more Latina than the Anglo-American agent, $F(1,42)=18.1, p<.001$, $\mathrm{n}^{2}=0.254$. The Anglo-American agent was perceived as slightly more American than the Latina agent was, although the difference was not significant, $F(1,42)=1.05$, n.s. Agents that spoke English were rated as significantly more American, $F(1,42)=6.98$, $\mathrm{p}=.012, \mathrm{y}^{2}=0.148$, while Spanish-speaking agents were rated as significantly more Latina, $F(1,42)=13.06, \mathrm{p}=.001, \mathrm{y}^{2}=0.184$. Participants were also significantly more 
likely to rate the agent as a member of their own culture if the agent's simulated culture was congruent with theirs, $F(1,42)=5.299, p=0.026, \mathrm{y}^{2}=0.114$.

Outcomes. We analyzed the data with a $2 \times 2 \times 2$ between-subjects MANOVA, using linguistic congruity, cultural congruity and need-for-cognition (split at the median) as independent variables, and persuasion (the difference between the scores of pre- and post- ranking tasks) and agent trustworthiness as dependent variables. A significant interaction effect of congruity and need-for-cognition was found on both persuasion, $\mathrm{F}(1,42)=5.537, \mathrm{p}=0.024, \quad \mathrm{p}^{2}=0.129$, and agent trustworthiness, $\mathrm{F}(1,42)=4.583$, $\mathrm{p}=0.039, \mathrm{y}^{2}=0.096$. Figure 3 shows this effect. A near significant main effect of needfor-cognition was also found on agent trustworthiness, $F(1,42)=4.070, p=0.051$, $\mathrm{y}^{2}=0.085$. There were no significant effects of linguistic congruity on outcomes.
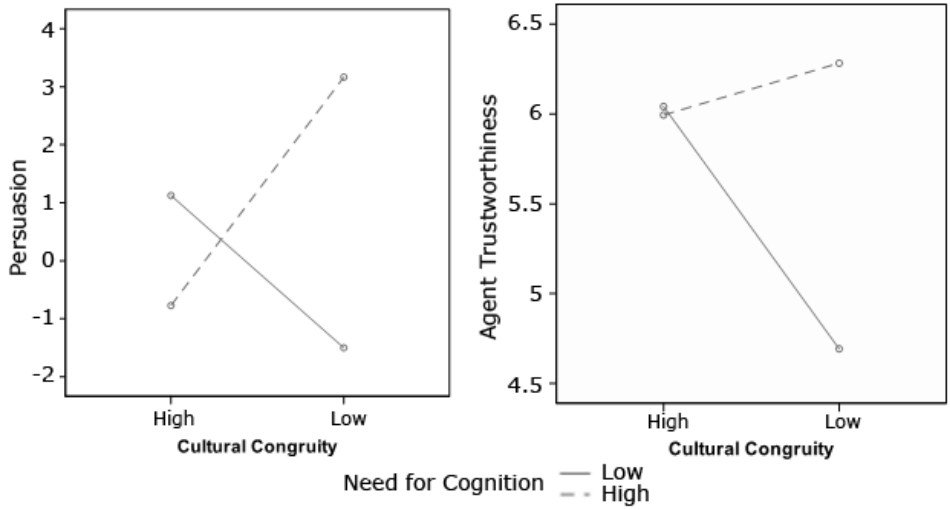

Fig. 3. Effect of need for cognition and cultural congruity on persuasion and agent trustworthiness

Many subjects mentioned that the agent's language strongly influenced their perception of the agent's cultural background. As an example, the following quote is from a Latino participant interacting with an English-speaking Latina agent.

"She seems more American, no but she said that... she came from Latin America right? Since she spoke English I'd say she's more Americanized than um... her original background. But she could be like me, you know, because I speak English and I'm a Hispanic. ... Yeah, I think she's more American because you know she spoke um... perfect English.” (Participant 33)

This indicates that an English-speaking agent with a Latino appearance is considered, to a large extent, a member of the American culture, or at least highly "Americanized". The Spanish-speaking Anglo-American agent was also considered as a Latina or as "neutral", as much as she was considered an American, regardless of her appearance.

Several Latino participants also recognized the Latina agent as from the Caribbean. This indicates that subtle cultural traits specific to one or a few Latin American countries, when implemented in the agent, can be easily picked up by a member of the Latino culture, and that "Latino" should not be treated as a unitary culture. 


\section{$5 \quad$ Discussion and Future Work}

Our findings suggest that participants who are predisposed to being influenced by peripheral cues tend to trust and be persuaded by an agent tailored to their culture more than by an agent from a different culture. Since most participants have been doing regular exercise for over six months, and many mentioned they already knew what the agent talked about, this is unlikely caused by an increase in the knowledge of physical activity, but rather because individuals taking the peripheral route to persuasion rely on environmental characteristics of the message delivered, and an agent resembling their in-group members is considered more credible and trustworthy, which is consistent with the Elaboration Likelihood Model. However, our findings also suggest that participants who are predisposed to process information deeply (i.e. high need for cognition) tend to be persuaded more by an agent from a different culture compared to one from their own culture. This may be explained by the fact that short-term engagement and relationship between speaker and hearer can reduce the impact of the speaker's persuasiveness on high-involvement topics (important topics that require thorough analysis) [16], since an interaction with an agent of high cultural congruity is presumably highly engaging and involving.

Linguistic tailoring had no effect on persuasion. This may be explained by the fact that participants were bilingual and their level of fluency in both languages was very high (all rated their proficiencies of both languages as 5 or higher on a 7-point scale).

Our results are from a single conversation between a user and a counseling agent, however, so one important direction of future research is to examine whether our results hold over repeated interactions. The correlation between persuasion and the agent's trustworthiness needs to be examined in greater detail. Future studies should be designed to examine trust as a mediating factor of persuasion and the interaction of cultural congruity and need for cognition. Our study also looked at the agent's appearance, nonverbal behaviors and the message delivered as a single cultural variable, but future research may investigate these aspects separately. Significant work also needs to be done on developing a systematic methodology for cultural tailoring of conversational agents, whether driven by a taxonomy such as Hofstede's cultural dimensions theory [17] or deeper knowledge of the norms and practices of the target culture.

Acknowledgments. We want to thank Juan Fernandez for administering the experiment, Daniel Schulman, Laura Pfeifer, Lazlo Ring, and the other members of the Relational Agents Group at Northeastern University for their help and insightful comments.

\section{References}

1. Hawkins, R.P., Kreuter, M., Resnicow, K., Fishbein, M., Dijkstra, A.: Understanding Tailoring in Communicating About Health. Health Educ. Res. 23, 454-466 (2008) 
2. Petty, R., Wegener, D.: Attitude Change: Multiple Roles for Persuasion Variables. The handbook of social psychology 1, 323-390 (1998)

3. Cacioppo, J., Petty, R., Feinstein, J., Blair, W., Jarvis, W.: Dispositional Differences in Cognitive Motivation: The Life and Times of Individuals Varying in Need for Cognition. Psychological Bulletin 119, 197-253 (1996)

4. Luna, D., Peracchio, L., de Juan, M.: The Impact of Language and Congruity on Persuasion in Multicultural E-marketing. Journal of Consumer Psychology 13 (1\&2), 41-50 (2003)

5. Rossen, B., Johnsen, K., Deladisma, A., Lind, S., Lok, B.: Virtual Humans Elicit Skin-Tone Bias Consistent with Real-World Skin-Tone Biases. IVA 2008, LNAI 5208, pp. 237-244. Springer (2008)

6. $\quad$ Endrass, B., Rehm, M., André, E.: Culture-Specific Communication Management For Virtual Agents. Proc. of 8th Int. Conf. on Autonomous Agents and Multiagent Systems (2009) 7. $\quad$ Endrass, B., Andre, E., Huang, L., Gratch, J.: A Data-Driven Approach to Culturespecific Communication Management Styles for Virtual Agents. Proc. of 9th Int. Conf. on Autonomous Agents and Multiagent Systems (2010)

8. Jan, D., Herrera, D., Martinovski, B., Novick, D., Traum, D.: A Computational Model of Culture-Specific Conversational Behavior. IVA 2007, LNAI 4722, pp. 45-56. Springer, (2007)

9. Zanbaka, C., Goolkasian, P., Hodges, L.: Can a Virtual Cat Persuade You?: The Role of Gender and Realism in Speaker Persuasiveness. Proceedings of the SIGCHI conference on Human Factors in computing systems, pp. 1153-1162. ACM (2006)

10. Katagiri, Y., Takahashi, T., Takeuchi, Y.: Social Persuasion in Human-Agent Interaction. Second IJCAI Workshop on Knowledge and Reasoning in Practical Dialogue Systems, pp. 64-69 (2001)

11. Schulman, D., Bickmore, T.: Persuading users through counseling dialogue with a conversational agent. Proceedings of the 4 th International Conference on Persuasive Technology (2009)

12. Lafferty, J., Eady, P.: The Desert Survival Problem. Experimental Learning Methods (1974)

13. Sue, D., Ivey, A., Pedersen, P.: A Theory of Multicultural Counseling and Therapy. Brooks/Cole Pacific Grove, Calif. (1996)

14. Albert, R., Ha, A.: Latino/Anglo-American Differences in Attributions to Situations Involving Touch and Silence. International Journal of Intercultural Relations 28, 253-280 (2004)

15. Wheeless, L., Grotz, J.: The Measurement of Trust and Its Relationship to SelfDisclosure. Human Communication Research 3, 250-257 (2006)

16. Dolinski, D., Nawrat, M., Rudak, I.: Dialogue Involvement as a Social Influence Technique. Personality and Social Psychology Bulletin 27, 1395 (2001)

17. Hofstede, G.: Culture's consequences: Comparing Values, Behaviors, Institutions, and Organizations Across Nations. Sage Pubns (2001) 